\title{
Ellagic Acid Enhances Antitumor Efficacy of Temozolomide in an in vitro Glioblastoma Model
}

\author{
Abdurrahman CETIN*, Burcu BILTEKIN² \\ ${ }^{1}$ Health Sciences University, Gazi Yasargil Education and Research Hospital, Department of Neurosurgery, Diyarbakir, Turkey \\ ${ }^{2}$ Istanbul University, Cerrahpasa Faculty of Medicine, Department of Histology and Embryology, Cerrahpasa, Istanbul, Turkey \\ Corresponding author: Abdurrahman CETIN acetin2147@gmail.com
}

\section{ABSTRACT}

AIM: To observe the effect of combining ellagic acid (EA), a natural phenol present in fruits and vegetables, and temozolomide (TMZ) on the proliferation and expression profile of $\mathrm{C} 6$ glioma cell line.

MATERIAL and METHODS: Rat C6 glioma cells were treated with 100- $\mu$ M EA combined with $100 \mu \mathrm{M}$ TMZ for 24, 48, and 72 hours (h). Cell proliferation and p53 and caspase-3 protein levels were evaluated using immunocytochemistry. Multi drug resistance 1 (MDR1), O6-methylguanine-DNA methyltransferase (MGMT), and apoptotic protein (caspase-3 and p53) expressions were assessed using reverse transcription polymerase chain reaction (RT-PCR).

RESULTS: EA combined with TMZ conspicuously reduced the cell viability at all incubation times $(p<0.001)$. EA significantly downregulated MGMT expression regardless of the presence of TMZ even at early hours $(p<0.001)$. The combination therapy reduced MDR1 expression only on $48 \mathrm{~h}$ in comparison with TMZ alone. EA alone upregulated caspase-3 at $48 \mathrm{~h}$ but upregulated p53 at 48 and $72 \mathrm{~h}$. The combined therapy enhanced the immunoreactivities of p53 and caspase-3 proteins independent of the treatment durations but not of the genes.

CONCLUSION: EA combined with TMZ may have a potential antiproliferative efficacy by inhibiting MGMT expression and activating apoptotic protein, p53 and caspase-3, expression.

KEYWORDS: Ellagic acid, Temozolomide, Glioma, MGMT, C6 glioma

ABBREVIATIONS: GBM: Glioblastoma, EA: Ellagic acid, TMZ: Temozolomide, MDR1: Multi drug resistance 1, MGMT: O6methylguanine -DNA methyltransferase, RT-PCR: Reverse transcription polymerase chain reaction, BrdU: 5-Bromo-2'-Deoxyuridine, Nf-kB: Nuclear factor kappa B

\section{INTRODUCTION}

$\mathrm{G}$ lioblastoma (GBM) is a common aggressive and malignant tumor that affects the central nervous system and has poor prognosis in children and adults (1). It generally spreads through the cerebrospinal fluid and metastasizes to normal brain tissues and the spinal cord, establishing a satellite tumor group around the primary tumor owing to its uncontrolled aggressive behavior and invasion ability (33). Although it can be managed by medical procedures such as surgery, radiation, and chemotherapy, the survival of patients with malignant gliomas has been reported between 14 weeks (no treatment) and 40-50 weeks (1). This is because of highgrade gliomas, which generally show local recurrence (17). Thus, the development of innovative therapeutic strategies and more effective agents is urgently necessary to prolong the survival and improve quality of life of patients (35). Combinations of new agents with basic chemotherapeutics, which are highly efficacious and safe, are required to optimize the efficacy of cancer treatment, resulting in a more suitable choice for chemotherapy. 
Combinations of chemical compounds, such as phenolic acids and flavonoids extracted from fruits and vegetables, may intensify the cytotoxic or antiproliferative effects of cancer therapeutic agents. Anatural phenolic compound, ellagic acid (EA), is found in strawberries, walnuts, cranberries, raspberries, pecans, pomegranates, and other plant foods (16). EA has been claimed to have anticarcinogenic properties, which are mediated via cell cycle arrest, tumor formation and growth inhibition, and apoptosis induction (4), or via angiogenesis suppression (18). EA is generally used as a dietary supplement to decrease or prevent the risk of cancer. However, the effect of its combination therapy with common chemotherapeutic agents used in glioma is still unclear.

The current clinical management of GBM includes surgical resection combined with radiotherapy and chemotherapy. A DNA-alkylating agent, temozolomide (TMZ) is commonly used as an chemotherapeutic agent (7), which is a secondgeneration oral ketamine that readily penetrates the bloodbrain barrier (34). TMZ has demonstrated its efficacy for GBM by attacking tumor cell DNA, damaging DNA by alkylation. However, multidrug resistance remains a primary obstacle in successfully treating GBM in humans $(21,34)$. Therefore, it is suggested that therapies targeting the metabolic adaptation mechanisms and the multidrug-resistant genes of tumor cells may provide a synergistic effect in TMZ therapy. In fact, a number of preclinical studies have indicated that TMZ, in combination with inhibitors that target invasion mechanisms and cellular metabolism, decelerated tumor progression $(7,34)$. To highlight the mechanism underlying the possible synergistic activity using EA, we investigated the effect of EA combined with $\mathrm{TMZ}$ on the drug resistance capacity, DNA repair mechanism, and apoptosis in C6 glioma cell line.

\section{MATERIAL and METHODS}

\section{Cell Culture}

C6 glioma cells obtained from the American Type Culture Collection were cultured at $37^{\circ} \mathrm{C}$ in $95 \%$ humidified air with $5 \% \mathrm{CO}_{2}$ in Dulbecco's Modified Eagle Medium, supplemented with $10 \%$ fetal bovine serum, $100 \mathrm{U} / \mathrm{mL}$ penicillin, and $100 \mu \mathrm{g} /$ $\mathrm{mL}$ streptomycin. The cells were subcultured every third day using trypsin. All concentrations are handled according to dose experiments as a range of 1,10 , and $100 \mu \mathrm{M}$ for both EA and $T M Z$, determined using the doses described in literature $(31,34)$. Accepted doses of EA (Sigma-Aldrich, E2250-10G,St. Louis, MO) and TMZ (Temozolid $20 \mathrm{mg}$, Dem İlaç, Istanbul, Turkey), both at $100-\mu \mathrm{M}$ dose, were added to the media and the cells were incubated for 24,48 , and 72 hours (h). The cells were divided into four groups for every incubation time-the control, TMZ, EA, and EA combined with TMZ (EA+TMZ). All experiments were repeated for at least three times.

\section{5-Bromo-2'-Deoxyuridine (BrdU) proliferation assay}

BrdU proliferation assay was performed, according to the literature, based on immunohistochemistry (24). BrdU was purchased from Santa Cruz Biotechnology (SC-32323; Santa Cruz, CA); Histostain-Plus Bulk Kit was from SensiTekScyTek Laboratories (Utah, USA). Mouse monoclonal anti-BrdU
(Bu20A, SC-20045; Santa Cruz Biotechnology, Santa Cruz, CA) was used as the primary antibody (1:200, overnight). The aminoethylcarbazole (AEC) chromogen (SensiTekScyTek Laboratories, Utah, USA) was then applied. BrdU labeling was assessed by two researchers, and the proliferation index was calculated by evaluating at least 3000 cells and scored as a number of positively stained cells/total number of cells counted (24).

\section{Immunohistochemistry}

C6 glioma cells cultured on cover slips were incubated for $24 \mathrm{~h}$; subsequently, groups of EA and TMZ were established. After 24-, 48-, and 72-h incubation periods, the experiments were ended at given durations and repeated three times. The cells were fixed with cold methanol for 5 minutes, and immunostaining was performed through indirect streptavidin immunoperoxidase method using an anti-polyvalent HRP Kit (SensiTekScyTek Laboratories, Utah, USA) to detect p53 and caspase-3 proteins. The cover slips were incubated overnight at $4^{\circ} \mathrm{C}$ with primary antibodies, namely, anti-p53 (orb136435; Biorbyt, California, USA) and anti-caspase-3 (active form, AB3623; Millipore, Darmstadt, Germany); these antibodies were diluted according to their protocols. The antigenantibody complex was subsequently visualized using the AEC Substrate Detection System (SensiTekScyTek Laboratories, Utah, USA). The intensity of immunoreactivity was evaluated semi-quantitatively using $\mathrm{H}$-SCORE analysis according to the literature (24).

\section{Expression Analysis}

Total RNA was extracted using Total RNA Purification Kit (Jena Bioscience, Germany) according to the kit protocol. cDNA was reverse-transcribed using the SCRIPT cDNA Synthesis Kit (Jena Bioscience, Germany), following the manufacturer's instructions. Real-time quantitative PCR (qPCR) was conducted in a CFX96 Touch (Bio-Rad, USA) machine using qPCR Green Master UNG (Jena Bioscience, Germany). Primer pairs were MDR1, F: 5'-CAGTTCATTCGCTCCTGACTAC-3' and R: 5'-CGTGCTGTAGCTGTCAATCT-3'; O6-methylguanineDNA methyltransferase (MGMT), F: 5'-GAAGCCTATTTCCACGAACCT-3' and R: 5'-CACCTGTCTGGTGAATGAATCT-3'; p53, F: 5'-ACATGACTGAGGTCGTGAGA-3' and R: 5'-GATTTCCTTCCACCCGGATAAG-3'; caspase-3, F: 5'-CTGACTGGAAAGCCGAAACT-3' and R: 5'-GTTCCACTGTCTGTCTCAATACC-3'; glyceraldehyde 3-phosphate dehydrogenase(GAPDH), F: 5'-GCAAGGATACTGAGAGCAAGAG-3'; and R: 5'-GGATGGAATTGTGAGGGAGATG-3'. After being normalized to GAPDH levels, the relative amount of MDR1, MGMT, p53, and caspase-3 transcripts in treated cells compared with controls were calculated as means \pm standard error of the mean (SEM).

\section{Statistical Analysis}

Semi-quantitative and quantitative data from all groups were evaluated statistically by GraphPad Instat version 3.06 program (GraphPad Inc, CA, USA). All data were presented as the mean \pm SEM. The mean of continuous variables was evaluated using the one way ANOVA, and variations between groups were compared using the Tukey-Kramer Multiple Comparison 
Test. $p<0.05, p<0.01$, and $p<0.001$ values were accepted as statistically significant.

\section{RESULTS}

Treatment with EA in combination with $T M Z$ enhances inhibition of cell proliferation

To determine whether EA could potentiate the inhibitory effects of TMZ on the proliferation of $\mathrm{C} 6$ glioma cells, we, first, semi-quantitatively analyzed the effects of EA combined with $\mathrm{TMZ}$ and $\mathrm{EA}$ without TMZ by using BrdU proliferation assay. As shown in Figure 1, there is a significant suppression of cell proliferation activity in the group treated with EA alone in a time-dependent manner as compared with the control groups $(p<0.001)$. Moreover, combined treatment with EA and TMZ enhanced TMZ-mediated inhibition of cell proliferation more significantly than treatment with $T M Z$ alone $(p<0.001)$, independently of treatment time.

EA affects the apoptotic proteins of p53 and caspase-3 at protein level in a time-dependent manner, regardless of the presence of TMZ

To determine the apoptotic effects of EA on glioma cells, p53 and caspase-3 expressions were investigated through qPCR (Figure 2), and their protein levels were investigated through immunohistochemistry (Figure 3 and Figure 4). At 24 h, TMZ significantly upregulated $p 53$ and caspase-3 expressions $(\mathrm{p}<0.001)$ in the cells; however, EA significantly altered $p 53$ expression only at $24 \mathrm{~h}(\mathrm{p}<0.01)$, only when combined with TMZ. In addition, EA with or without TMZ increased the reactivities of both proteins at all incubation times $(p<0.001)$. Although slight increases in p53 expression at 48 and

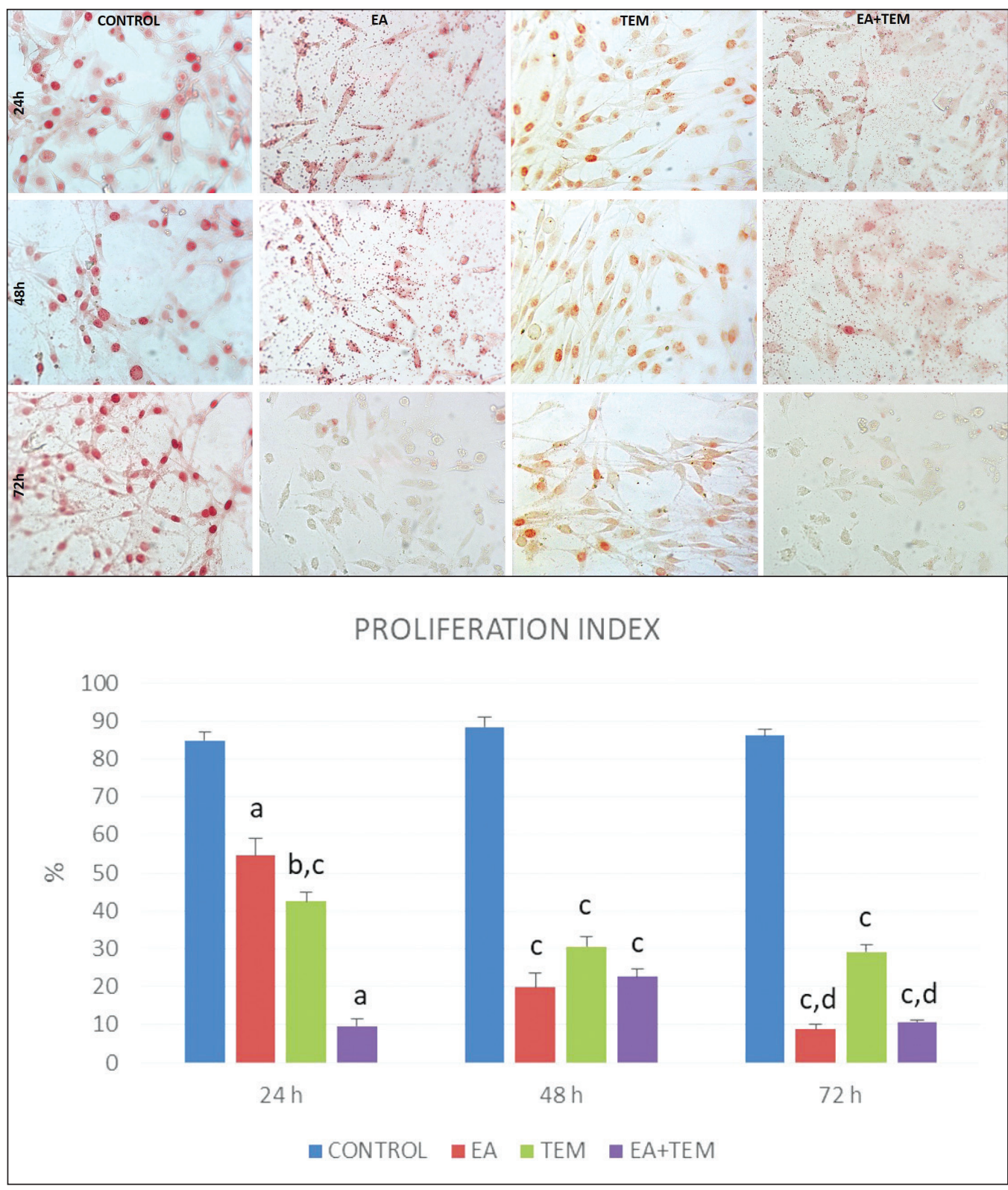

Figure 1: Microphotographs and graphical presentation of BrdU proliferation assay on C6 glioma cells treated with ellagic acid (EA) and temozolomide (TMZ); magnification: 400X.

a) $p<0.001$ vs. all groups;

b) $p<0.01$ vs. EA group; c) $p<0.001$ vs. control group; d) $\mathrm{p}<0.001$ vs. TMZ group. 
$72 \mathrm{~h}$ in the combination groups were seen as compared with the control group, the differences were not significant. EA alone succeeded to upregulate p53 expression at late hours $(p<0.001)$, probably by mediating post-translational modifications. EA alone demonstrated a similar modification of caspase-3 expression during the $48-h$ treatment $(p<0.01)$.

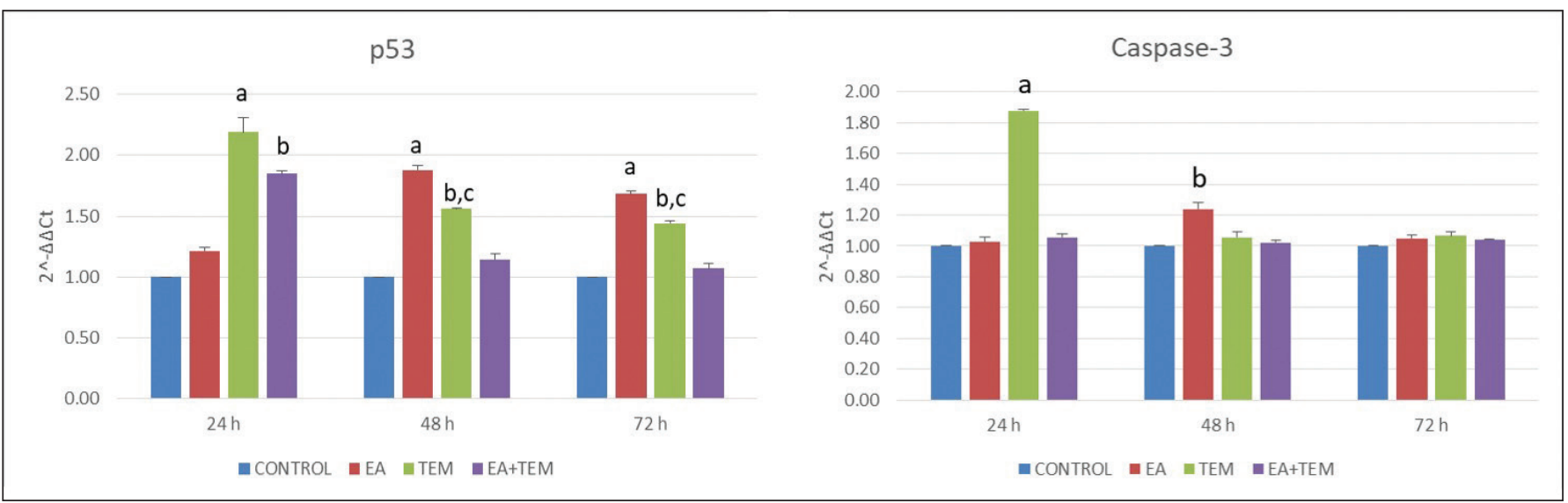

Figure 2: Graphical presentation of RT-PCR results of $p 53$ and caspase-3 expressions on C6 glioma cells treated with ellagic acid (EA) and temozolomide (TMZ). a) $p<0.001$ vs. all groups; b) $p<0.01$ vs. control group; $\mathbf{c}) p<0.01$ vs. EA+TMZ group.

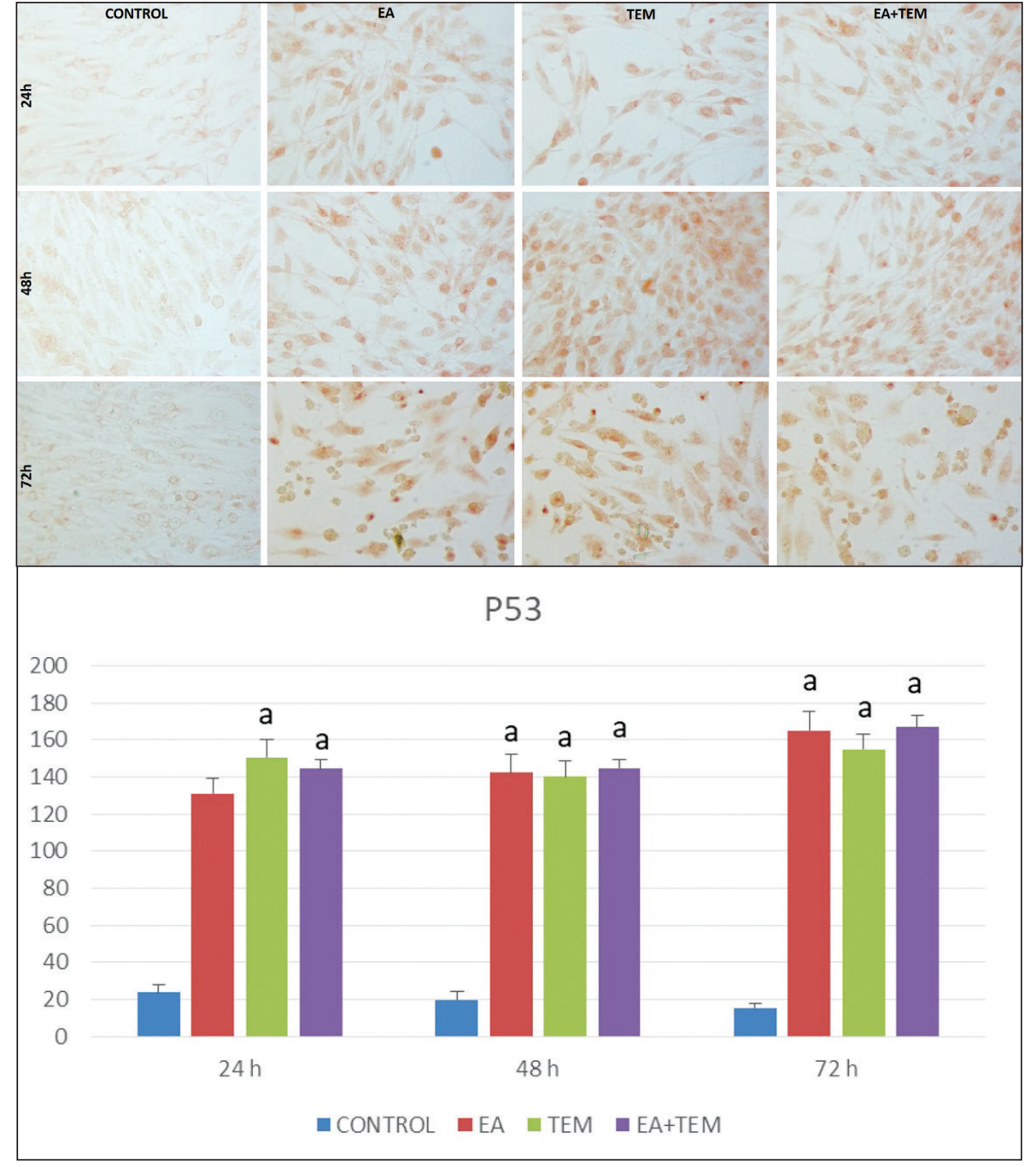

Figure 3: Microphotographs and graphical presentation of p53 immunohistochemical results of $\mathrm{C} 6$ glioma cells treated with ellagic acid (EA) and temozolomide (TMZ); magnification: 400X. a) $p<0.0001$ vs. control group; b) $p<0.05$ vs. TMZ group. 


\section{EA combined with TMZ failed to downregulate MDR1 expression (ABCB1)}

As a modulatory gene in drug resistance, MDR1 (ABCB1) is often involved in the nonresponsive features of glioma cells (9). The present study investigated whether EA in combination with TMZ affected MDR1 expression TMZ. The results indicated that treatment with $E A$ alone and in combination with TMZ failed to reduce MDR1 expression TMZ at longer incubations (Figure 5). In fact, its expression was upregulated significantly by EA only at $48 \mathrm{~h}(\mathrm{p}<0.001)$, where as addition of $T M Z$ suppressed this boosting effect and reduced its expression in the control levels. TMZ alone also showed an increased MDR1 expression $(p<0.001)$ at $48 \mathrm{~h}$ but not at 72 $\mathrm{h}$, which suggests that only combined application of EA with TMZ may have an inhibitory effect in the multidrug resistance capacity of the $\mathrm{C} 6$ glioma cells at the early hours.
Ellagic acid downregulates the MGMT expression in a time-dependent manner regardless of the presence of TMZ

MGMT expression in EA-treated C6 glioma cells were investigated to determine whether MGMT expression, which is highly encountered in solid types of cancer and has proven to remove the drug-induced cytotoxic O6-alkylguanine DNA adducts, is regulated by EA. At $24 \mathrm{~h}$, EA suppressed TMZ to induce MGMT overexpression $(p<0.001)$ (Figure 6). Interestingly, at 48 and $72 \mathrm{~h}$ of incubation, EA down regulated MGMT expression dramatically whether combined with TMZ or not $(p<0.001)$, suggesting that the antiproliferative properties of EA may be related to the modulatory effect of EA on MGMT.

\section{DISCUSSION}

Up to $95 \%$ of patients having GBM have been defeated by

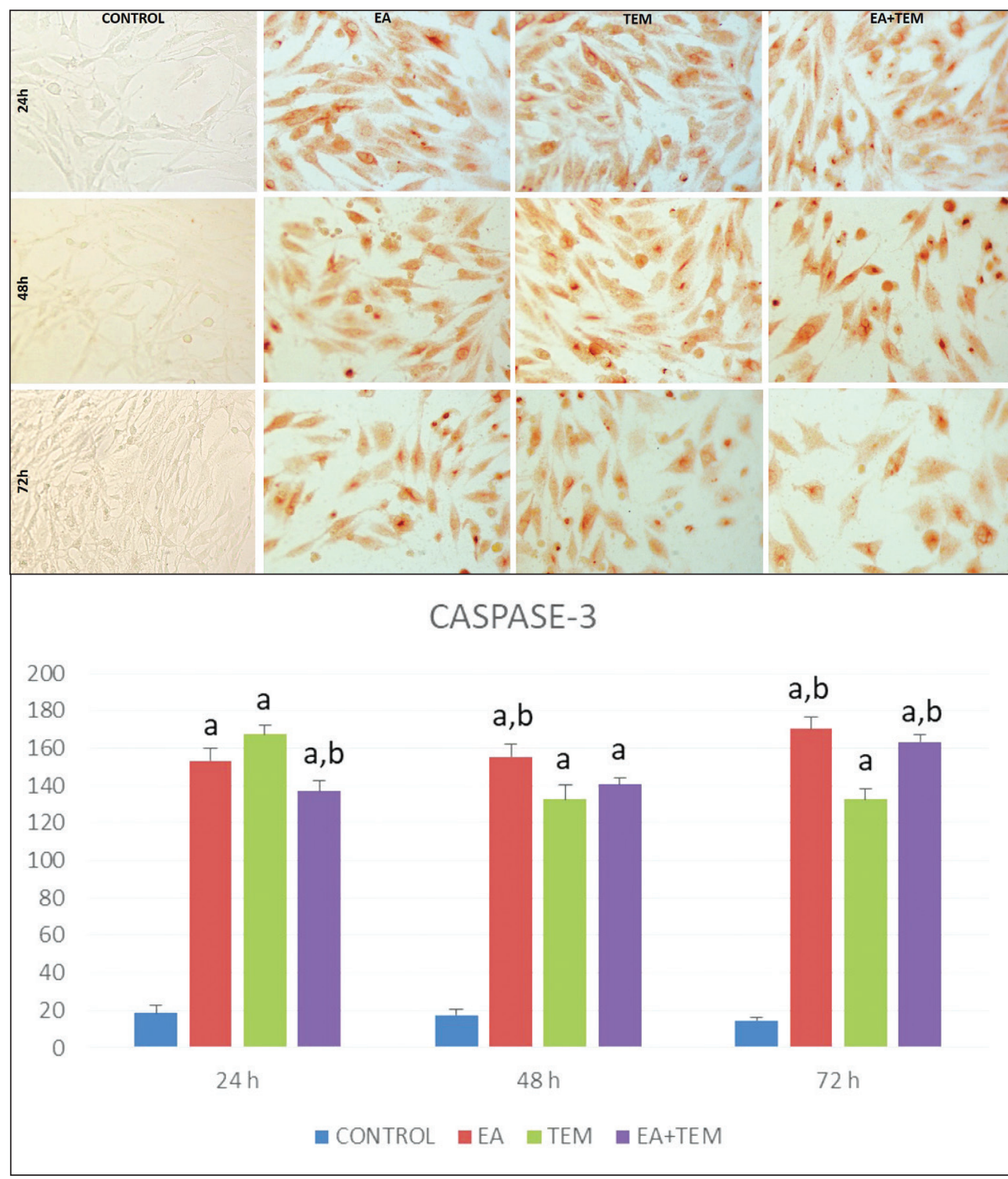

Figure 4: Microphotographs and graphical presentation of caspase- 3 immunohistochemical results of $\mathrm{C} 6$ glioma cells treated with ellagic acid (EA) and temozolomide (TMZ); magnification: 400X. a) $p<0.0001$ vs. control group; b) $\mathrm{p}<0.05$ vs. TMZ group. 

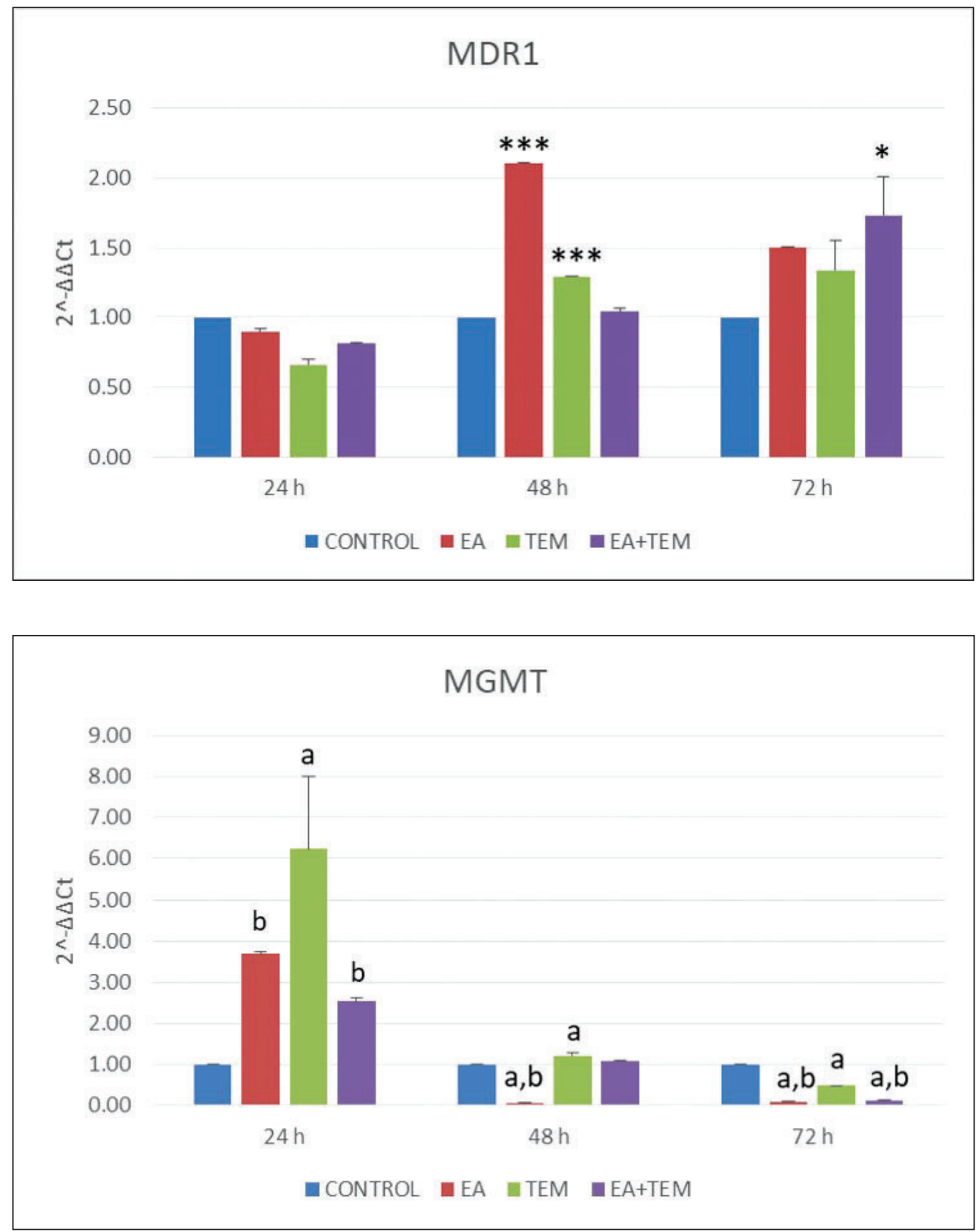

Figure 5: Graphical presentation of RT-PCR results of MDR1expression on C6 glioma cells treated with ellagic acid (EA) and temozolomide (TMZ) ${ }^{*} \mathrm{p}<0.05$ vs. control group; ${ }^{* * *} p<0.001$ vs. control group
Figure 6: Graphical presentation of RT-PCR results of MGMT expression on $\mathrm{C} 6$ glioma cells treated with ellagic acid (EA) and temozolomide (TMZ)

a) $p<0.001$ vs. control group; b) $\mathrm{p}<0.001$ vs. TMZ group the disease and died within 5 years after initial diagnosis. Investigating novel strategies to treat GBM seems to be crucial, including targeting the metabolic adaptation mechanisms and utilizing combination therapies of chemotherapeutic drugs with inhibitors that target drug-resistant mechanisms or with various anti-inflammatory, antineoplastic, apoptotic, or toxic agents (5). Multiple targets for the emergence of drug-resistant tumors should aim to reduce the toxicity of chemotherapy and improve its efficacy (28). Research conducted on the synergistic properties of antitumor drugs gain attention so as to regulate apoptotic pathways, reverse multidrug resistance, inhibit tumor migration and invasion, and prevent angiogenesis system $(3,6,14)$. In patient with
GBM, long-term use of $\mathrm{TMZ}$ is disadvantageous due to development of drug resistance, resulting in a reduced efficacy of TMZ in time (20). Thus, it is necessary to discover new therapeutic approaches in overcoming this obstacle such as developing combination therapies of naturally occurring dietary polyphenolic compound like EA. EA is known to have antioxidant, antifibrotic, and anticarcinogenic properties (27). Hence, we revealed the combining effect of EA with $T M Z$ on the proliferation and expression profile of C6 glioma cell line.

Various studies have revealed that EA plays a significant role in inhibiting cell proliferation, preventing metastasis, and controlling tumor cell invasion. In a study by Wang et al., EA has shown to inhibit the cell viability, proliferation, and 
invasion of human GBM cells, suggesting that the cell cycle arrest and DNA damage induced by EA may promote the inhibitory effects (30). EA has been demonstrated to reduce the prevalence of a variety of carcinogen-induced tumors (19), including distinctive anticarcinogenic effects in several types of cancers $(4,19,29-31)$. Wang et al. also indicated that EA dramatically reduced the cell viability of GBM cells at the range of 25-200 $\mu \mathrm{M}$ doses, suggesting its antiproliferative efficacy. Most importantly, EA downregulated anti-apoptotic protein (survivin and $\mathrm{Bcl}-2$ ) expressions but elevated caspase-3 and pro-apoptotic protein Bax expressions in a dose-dependent manner (30). Another study showed that EA induced G1 arrest and apoptosis by p53-mediated activation in cells (10) while it inhibits angiogenesis by inactivating metalloproteinases (11). Additionally, TMZ was shown to significantly affect the apoptotic signal pathways in $\mathrm{C} 6$ cells by synergistic alteration with an antibiotic, by regulating $\mathrm{Bax}$ and procaspase- 3 expressions (13). In the present study, TMZ significantly upregulated p53 and caspase- 3 expressions at $24 \mathrm{~h}$ and increased the immunoreactivities of these proteins in cells at all incubation times. However, EA showed its activatory effect on $p 53$ and caspase-3 expressions at longer incubation times, where as activatory effect on p53 protein levels was shown at all incubation durations. Combination with TMZ was only successful in inducing apoptotic p53 expression at $24 \mathrm{~h}$ but not caspase-3 expression and other incubation times, although the protein levels of both were significantly elevated, suggesting a modulatory role of EA in the post-translational modifications.

More than $90 \%$ of treatment failure in aggressive metastatic cancers like GBM is due to drug resistance and multiple mechanisms $(25,26)$. MDR pathways may be induced by elevated release of the drug from the cells to extracellular matrix, thereby reducing drug absorption in cancer cells (22). MDR characterized by increased $p$-glycoprotein expression may be a remarkable obstacle in treating cancer (8), as this is related to drug nonresponse in malignant tumors. In vitro studies have shown that untreated GBM principally express MDR1 even if they are at least partially chemosensitive (12). One of them tested the chemosensitivity of GBM to some antineoplastic drugs on cultured tissue; it demonstrated MDR1 expression in 16 sensitive and five highly resistant GBM cells (12). All of the 21 tumors identically expressed $p$-glycoprotein, suggesting that untreated GBM mostly express $p$-glycoprotein, which results in the existence of cell populations with early drug resistance in these tumors. This phenomenon may explain the disappointing overall long-term efficacy of chemotherapy. In the present study, we investigated whether EA in combination with TMZ modulates MDR1 expression profile in C6 glioma cells. We found that EA failed to reduce MDR1 expression alone or in combination with TMZ during longer incubation periods. In fact, its expression was upregulated significantly by EA only at $48 \mathrm{~h}$, whereas addition of TMZ suppressed this boosting effect and reduced its expression, suggesting that only combined therapy of EA and TMZ may have an inhibitory effect at early hours in multidrug resistance capacity of the C6 glioma cells. High expression levels at 48 and $72 \mathrm{~h}$ is probably due to post-translational modulation by EA or adaptation of cells tolong incubation with EA, which may lead to drug resistance. This may be considered as a limitation of the present study.

GBM is resistant to some chemotherapeutic drugs because of the MGMT gene in glioma cells (23), which is highly expressed in solid types of cancer, resulting in the removal of the druginduced cytotoxic O6-alkylguanine DNA adducts. This unique DNA repair protein, MGMT, has the ability to prevent the formation of DNA interstrand crosslinks in majority of human tumors, which are considered to be the critical lethal lesion induced by these drugs. The methylation status of MGMT promoter is a crucial indicator of the prognosis of GBM (2). There is an immediate need to develop well-tolerated drugs with the capacity to reverse drug resistance. In fact, various agents targeting drug resistance, with the capacity to mediate MGMT expression, are actively being studied. Therefore, we focused on the combination treatments of TMZ and EA in order to inhibit unforeseen DNA repair by mediating MGMT expression. We found that EA suppressed TMZ-induced MGMT over expression in a time-dependent manner, suggesting a modulatory effect of EA in combination with TMZ on MGMT expression. Additionally, since treatment of EA alone was able to inhibit MGMT expression, it may be considered that EA is able to show its antiproliferative feature by altering the DNA repair mechanism in C6 glioma cells.

Tumor cell invasion and metastasis result in an advanced tumor progression depending on the destruction to cellcell and cell-matrix adhesion. Signaling pathways of some regulatory proteins like matrix metalloproteases (MMPs) and transcription factors like nuclear factor kappa B (NF-kB) are known to play crucial roles in tumor development through the transcriptional regulation of genes associated with tumor growth, invasion, and metastasis $(13,32)$. Blocking the transcriptional activity of these proteins could inhibit glioma cell invasion; not being able to show a blocking effect of EA on one of the transcriptional and regulatory factor proteins in glioma cells is a limitation of our study. In a study by Luo et al., it was shown that down regulating a long non-coding RNA, namely, IncRNA UBE2CP3, could suppress glioma growth by regulating MMP9 protein expression in vitro and in vivo. They also hypothesized that IncRNA UBE2CP3 could affect the NFkB-MMP9 signaling pathway by mediating tumor necrosis factor receptor-associated factor 3 (TRAF3) interacting protein 2 , which is an upstream regulator of both IKK/NF-kB and JNK/AP-1 (15). Thus, in the future, we may focus on NF-kBMMP9 signaling pathway to show the relationship between modulatory and antiproliferative role of EA on glioma cells.

\section{- CONCLUSION}

Taken together, the present study suggests that EA has antiproliferative and apoptotic activity in C6 glioma cell line that is mediated through the activation of p53 expression and the inhibition of MGMT expression. Long-term treatment with EA combined with TMZ may have an inhibitory effect on multidrug resistance capacity of the glioma cells and have a suppressing effect on DNA repair activity induced by $T M Z$ in a time-dependent manner. This suggests a modulatory effect of 
EA in combination with $T M Z$ in cancer therapy. For including EA in the drug development and treatment protocols, more detailed and in vivo studies are required. Moreover, to develop a novel chemotherapeutic strategy to sensitize cancer cells to $\mathrm{TMZ}$, the drug resistance of combination therapies involving chemotherapeutic agents in the treatment of GBM should be resolved.

\section{- REFERENCES}

1. Avgeropoulos NG, Batchelor T: New treatment strategies for malignant gliomas. Oncologist 4:209-224, 1999

2. Brandes AA, Franceschi E, Tosoni A, Blatt V, Pession A, Tallini G, Bertorelle R, Bartolini S, Calbucci F, Andreoli A, Frezza G, Leonardi M, Spagnolli F, Ermani M: MGMT promoter methylation status can predict the incidence and outcome of pseudoprogression after concomitant radiochemotherapy in newly diagnosed glioblastoma patients. J ClinOncol 26(13): 2192-2197, 2008

3. Domvri K, Zarogoulidis K, Zogas N, Zarogoulidis P, Petanidis S, Porpodis K, Kioseoglou E, Hohenforst-Schmidt W: Potential synergistic effect of phosphodiesterase inhibitors with chemotherapy in lung cancer. J Cancer 8(18):3648-3656, 2017

4. Edderkaoui M, Odinokova I, Ohno I, Gukovsky I, Go VL, Pandol SJ, Gukovskaya AS: Ellagic acid induces apoptosis through inhibition of nuclear factor kappa B in pancreatic cancer cells. World J Gastroenterol 14(23):3672-3680, 2008

5. Hanif F, Muzaffar K, Perveen K, Malhi SM, Simjee ShU: Glioblastoma multiforme: A review of its epidemiology and pathogenesis through clinical presentation and treatment. Asian Pac J Cancer Prev 18(1):3-9, 2017

6. He Z, Xiao X, Li S, Guo Y, Huang Q, Shi X, Wang X, Liu Y: Oridonin induces apoptosis and reverses drug resistance in cisplatin resistant human gastric cancer cells. Oncol Lett 14(2):2499-2504, 2017

7. Hegi ME, Diserens AC, Gorlia T, Hamou MF, de Tribolet N, Weller M, Kros JM, Hainfellner JA, Mason W, Mariani L, Bromberg JE, Hau P, Mirimanoff RO, Cairncross JG, Janzer RC, Stupp R: MGMT gene silencing and benefit from temozolomide in glioblastoma. N Engl J Med 352(10):997-1003, 2005

8. Hermann DM, Kilic E, Spudich A, Kramer SD, WunderliAllenspach $\mathrm{H}$, Bassetti CL: Role of drug efflux carriers in the healthy and diseased brain. Ann Neurol 60:489-498, 2006

9. Hodges LM, Markova SM, Chinn LW, Gow JM, Kroetz DL, Klein TE, Altman RB: Very important pharmacogene summary: ABCB1 (MDR1, P-glycoprotein). Pharmacogenet Genomics 21(3):152-161, 2011

10. Khan N, Afaq F, Mukhtar H: Apoptosis by dietary factors: The suicide solution for delaying cancer growth. Carcinogenesis 28(2):233-239, 2007

11. Labrecque L, Lamy S, Chapus A, Mihoubi S, Durocher $Y$, Cass B, Bojanowski MW, Gingras D, Béliveau R: Combined inhibition of PDGF and VEGF receptors by ellagic acid, a dietary-derived phenolic compound. Carcinogenesis 26(4):821-826, 2005
12. Leweke F, Damian MS, Schindler C, Schachenmayr W: Multidrug resistance in glioblastoma. Chemosensitivity testing and immunohistochemical demonstration of P-glycoprotein. Pathol Res Pract 194(3):149-155, 1998

13. Li X, Ling N, Bai Y, Dong W, Hui GZ, Liu D, Zhao J, Hu J: MiR16-1 plays a role in reducing migration and invasion of glioma cells. Anat Rec (Hoboken) 296(3):427-432, 2013

14. Li XQ, Ouyang ZG, Zhang SH, Liu H, Shang Y, Li Y, Zhen YS: Synergy of enediyne antibiotic lidamycin and temozolomide in suppressing glioma growth with potentiated apoptosis induction. J Neurooncol 119(1):91-100, 2014

15. Luo Z, Pan J, Ding Y, Zhang YS, Zeng Y: The function and clinical relevance of IncRNA UBE2CP3-001 in human gliomas. Arch Med Sci 14(6):1308-1320, 2018

16. Mertens-Talcott SU, Talcott ST, Percival SS: Low concentrations of quercetin and ellagic acid synergistically influence proliferation, cytotoxicity and apoptosis in MOLT-4 human leukemia cells. Journal of Nutrition133:2669-2674, 2003

17. Moses MA, BremH, Langer R: Advancing the field of drug delivery: Taking aim at cancer. Cancer Cell 4:337-341, 2003

18. Narayanan BA, Geoffroy $O$, Willingham MC, Re GG, Nixon DW: p53/p21(WAF1/ CIP1) expression and its possible role in G1 arrest and apoptosis in ellagic acid treated cancer cells. Cancer Letters 136:215-221,1999

19. Nijhoff WA, Peters WH: Quantification of induction of rat oesophageal, gastric and pancreatic glutathione and glutathione S-transferases by dietary anticarcinogens. Carcinogenesis 15(9):1769-1772,1994

20. Pinheiro R, Braga C, Santos G, Bronze MR, Perry MJ, Moreira $R$, Brites D, Falcão AS: Targeting gliomas: Can a new alkylating hybrid compound make a difference? ACS Chem Neurosci 8(1):50-59, 2017

21. Salma S, Djan I, Bjelan M, Vulekovic P, Novakovic M, Vidovic V, Lucic M: Benefit and outcome of using temozolomidebased chemoradiotherapy followed by temozolomide alone for glioblastoma in clinical practice. J BUON 22(5):1233-1239, 2017

22. Sampath D, Cortes J, Estrov Z, Du M, Shi Z, Andreeff M, Gandhi V, Plunkett W: Pharmacodynamics of cytarabine alone and in combination with 7-hydroxystaurosporine (UCN-01) in AML blasts in vitro and during a clinical trial. Blood 107(6): 2517-2524, 2006

23. Sarkaria JN, Kitange GJ, James CD, Plummer R, Calvert $\mathrm{H}$, Weller M, Wick W: Mechanisms of chemoresistance to alkylating agents in malignant glioma. Clin Cancer Res 14: 2900-2908, 2008

24. Soydas T, Yaprak Sarac E, Cinar S, Dogan S, Solakoglu S, Tuncdemir M, Kanigur Sultuybek G: The protective effects of metformin in an in vitro model of aging 3T3 fibroblast under the high glucose conditions. J Physiol Biochem 74(2):273281, 2018

25. Takara K, Sakaeda T, Okumura K: An update on overcoming MDR1-mediated multidrug resistance in cancer chemotherapy. Curr Pharm Des 12:273-286, 2006

26. Thomas $\mathrm{H}$, Coley HM: Overcoming multidrug resistance in cancer: An update on the clinical strategy of inhibiting p-glycoprotein. Cancer Control 10:159-165, 2003 
27. Thresiamma KC, Kuttan R: Inhibition of liver fibrosis by ellagic acid. Indian J Physiol Pharmacol 40(4):363-366, 1996

28. Tortora G, Bianco R, Daniele G, Ciardiello F, McCubrey JA, Ricciardi MR, Ciuffreda L, Cognetti F, Tafuri A, Milella M: Overcoming resistance to molecularly targeted anticancer therapies: Rational drug combinations based on EGFR and MAPK inhibition for solid tumours and haematologic malignancies. Drug Resist Updat 10(3):81-100, 2007

29. Umesalma S, Sudhandiran G: Ellagic acid prevents rat colon carcinogenesis induced by 1, 2 dimethyl hydrazine through inhibition of AKT-phosphoinositide-3 kinase pathway. Eur J Pharmacol 660:249-258, 2011

30. Wang D, Chen Q, Liu B, Li Y, Tan Y, Yang B: Ellagic acid inhibits proliferation and induces apoptosis in human glioblastoma cells. Acta Cir Bras 31(2):143-149, 2016

31. Wang D, Chen Q, Tan Y, Liu B, Liu C: Ellagic acid inhibits human glioblastoma growthin vitro and in vivo. Oncol Rep 37(2):1084-1092, 2017
32. Wild-Bode C, Weller M, Wick W: Molecular determinants of glioma cell migration and invasion. J Neurosurg 94:978-984, 2001

33. Zhang H, Ma L, Wang Q, Zheng X, Wu C, Xu BN: Role of magnetic resonance spectroscopy for the differentiation of recurrent glioma from radiation necrosis: A systematic review and meta-analysis. Eur J Radiol 83:2181-2189, 2014

34. Zhang R, Saito R, Shibahara I, Sugiyama S, Kanamori M, Sonoda Y, Tominaga T: Temozolomide reverses doxorubicin resistance by inhibiting P-glycoprotein in malignant glioma cells. J Neurooncol 126(2):235-242, 2016

35. Zhao J, Zhu J, Lv X, Xing J, Liu S, Chen C, Xu Y: Curcumin potentiates the potent antitumor activity of $\mathrm{ACNU}$ against glioblastoma by suppressing the PI3K/AKT and NF-KB/COX2 signaling pathways. Onco Targets Ther 10:5471-5482, 2017 